\title{
Current Minimum in Differential Pulse Polarography
}

\author{
Marina Zelić, ${ }^{*}$ Ivanka Pižeta, and Dijana Jadreško \\ Division for Marine and Environmental Research, Ruđer Bošković Institute, POB 180, 10002 Zagreb, Croatia \\ RECEIVED JANUARY 26, 2012; REVISED MAY 17, 2012; ACCEPTED JULY 16, 2012
}

\begin{abstract}
Current minimum, which sometimes appears as a part of the net response in differential pulse polarography (DPP), was studied in systems characterized by a pronounced IR drop or reactant adsorption. Experimental results obtained on a static mercury drop electrode (SMDE) clearly indicate that this effect is highly influenced not only by solution resistance (or intentionally added resistors) and reactant concentration, but also by both timing parameters (drop time, pulse duration) and electrode surface area. Presentation of the net response along with its components, demonstrates that the current minimum originates from the maximum on dc component, minimum on pulse component or both. In practice, DPP minimum, obtained in measurements with a SMDE, can be treated as an additional diagnostic parameter for the recognition of reactant adsorption or poor experimental conditions (i.e. high resistance within electrode system or low conductivity of the electrolyte medium). (doi: 10.5562/cca2054)
\end{abstract}

Keywords: current minimum; IR drop; reactant adsorption; components of the net response; differential pulse polarography

\section{INTRODUCTION}

In polarography/voltammetry of simple redox processes, either sigmoidal or bell shaped current - potential curves are generally obtained; which type of curve manifests depends on the applied working electrode and/or measuring technique. ${ }^{1-3}$ In the former case, when so called waves are formed, the response could be complicated by a maximum (i.e. peak) that starts as a prolonged ascending part of the signal and ends at its limiting current. Exceptionally, as in the case of tellurium(IV) reduction, the extreme is located between two segments of the plateau. ${ }^{4}$

In normal pulse polarography (NPP), the maximum usually reflects reactant adsorption and can be used as a source of information about the process of interest. ${ }^{5-7}$ In direct current polarography the situation is more complicated $^{1,8-10}$ because maxima of at least three different kinds are possible. The current maximum is usually described in terms of the electrolyte circulation near electrode surface and is not easily simulated. Its suppression or even elimination is often achieved by addition of surface active substances to the electrolyte solution. ${ }^{1}$ Therefore older polarographic measurements were performed in the presence of dissolved gelatin or some other surfactant.

During the last three decades, i.e. after substitution of dropping mercury electrodes (DME) with static mercury drop electrodes (SMDE), maxima in dc polarogra- phy of simple redox processes virtually disappeared and application of their suppressors was completely abandoned. NPP maxima, however, remained preserved, due to their different origin.

Not only is there more or less pronounced maximum, but also a minimum sometimes appears on the plateau of a dc wave, ${ }^{11-13}$ whose origin could be found in surface processes although some other explanations are also possible.

Bell shaped signals generally do not "suffer" from additional extremes, however a minimum that sometimes appears on the basic current, immediately after the peak in differential pulse polarography (DPP) is known. ${ }^{12,14-18}$ Here, for the first time we observed this minimum during the study of lead(II) in chloride medi$\mathrm{um}^{19}$ as a possible result of reactant (i.e. $\mathrm{PbCl}_{3}{ }^{-}$) adsorption at the mercury drop. According to subsequent measurements, this effect was a consequence of some resistance within the electrode system. ${ }^{17}$ (Lead(II) adsorption from chloride media is too weak to be "seen" by voltammetry even when combination of a concentrated electrolyte solution, i.e. low water activity and a sensitive technique is applied.) After careful cleaning of SMDE, the minimum was not visible any more, but could be provoked again by addition of resistors between the working electrode and the input of the instrument. Under such conditions (in addition to the appearance of DPP minimum) the reduction signal be-

\footnotetext{
* Author to whom correspondence should be addressed. (E-mail: zelic@irb.hr)
} 
comes lower, wider and shifted to the more negative potentials. An attempt to simulate the influence of the IR drop on DPP response (starting from expressions given in Ref. 20) really gave the signals, changed in the mentioned way. Only the minimum was lacking.

In our and other previous articles, examples of such minima were trivialized or even ignored as other problems seemed more important. The DPP minimum has been used for the analytical determination of polysulfides ${ }^{12}$ for example, and in this article this effect will be dealt with in more detail. Our own dc and DPP results will be presented along with the literature data in attempt to recognize the origin of the whole effect and its possible applications.

\section{EXPERIMENTAL}

All solutions were prepared from reagent grade chemicals and deionized water. Before each measurement the aqueous medium in the polarographic cell was deaerated for 15 minutes by high purity nitrogen. DPP and dc signals were obtained by means of the static mercury drop electrode (model PAR 303 from Princeton Applied Research or 663 VA stand from Metrohm). Platinum wire served as a counter electrode and all potentials were defined with respect to the $\mathrm{Ag} / \mathrm{AgCl}$ reference electrode filled with saturated $\mathrm{NaCl}$ solution (PAR 303) or $3 \mathrm{~mol} / \mathrm{L} \mathrm{KCl}(663$ VA stand). In the latter case, an electrolyte bridge with 3 $\mathrm{mol} / \mathrm{L} \mathrm{NaCl}$ was added in order to prevent formation of the poorly soluble $\mathrm{KClO}_{4}$ in the frit during measurements in perchlorate medium. Through the corresponding IME unit, the electrode assembly was connected to the instrument ( $\mu$ Autolab from Eco Chemie, Utrecht, The Netherlands). Two different versions of the software were used, because only in such a way could all potential/timing parameters of interest be applied.

Measurements in the $\mathrm{Cd}^{2+}-\mathrm{I}^{-}$system were performed at three different ionic strengths $(1,4$ and 6 $\mathrm{mol} / \mathrm{L}$ ), maintained with $\mathrm{NaClO}_{4}$, in the presence of $0.001 \mathrm{~mol} / \mathrm{L} \mathrm{HClO}_{4}$. In each individual case, the iodide level was chosen so as to produce the highest possible relative concentration of the surface active complex $\mathrm{CdI}_{2}$. Two different metal concentrations that gave highly pronounced adsorption effects, i.e. completely covered electrode surface were applied at each ionic strength, in accordance with previous results. ${ }^{18}$ Laboratory temperature was kept at $24 \pm 1{ }^{\circ} \mathrm{C}$.

\section{RESULTS AND DISCUSSION}

\section{IR Drop}

Taking into account that each DPP signal can be presented in terms of its components (which result from the current sampling at the end of pulse and prepulse peri-

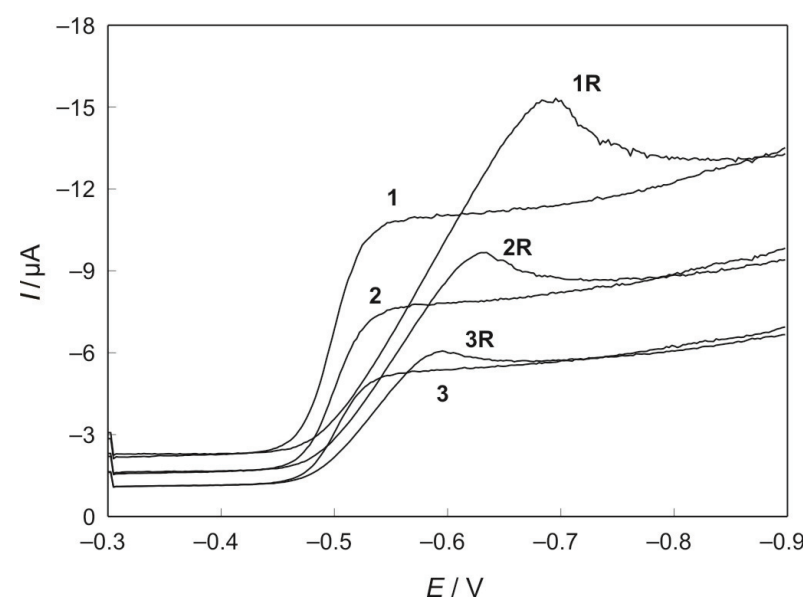

Figure 1. Sampled current polarograms of $1.4 \mathrm{mmol} / \mathrm{L} \mathrm{Cd}^{2+}$ in $0.8 \mathrm{~mol} / \mathrm{L} \mathrm{H}_{2} \mathrm{SO}_{4}$ recorded at drop times of 0.3 (1), 0.5 (2) and 1 (3) $\mathrm{s}$ in the absence of added resistance and the same responses obtained after addition of a $10 \mathrm{k} \Omega$ resistor (R) between working electrode and input of the instrument $(1 \mathrm{R}, 2 \mathrm{R}$, 3R). Electrode: PAR 303; mercury surface area: $1.68 \mathrm{~mm}^{2}$.

ods, respectively) ${ }^{15,21}$ insight into their shapes could give some information about the "formal" origin of the minimum that appears as an extension of the reduction signal, i.e. an inverse peak. Even when the applied instrument/software does not offer such a possibility it is useful to record the dc wave in addition to the net DPP signal, as the former is very similar to the wave component of the net response. Strictly speaking, drop time in such a case should be equal to the difference between drop time $t_{\mathrm{d}}$ and pulse duration $t_{\mathrm{p}}$ in the corresponding DPP experiment, although for qualitative conclusions measurements at the same drop time could be acceptable. From the resulting signals it becomes obvious that (in the simplest case) the DPP minimum appears as a consequence of the maximum on its dc component. In other words, for a deeper understanding of the whole effect, all factors that could induce formation of the polarographic maximum on dc wave (while using SMDE or DME) are important. One of them is the inner resistance within the measuring system. When a known resistor is intentionally added between the working electrode and the input of the current/voltage converter of the potentiostat, the originally simple dc wave of $\mathrm{Cd}^{2+}$ reduction in a relatively inert medium $(0.8 \mathrm{~mol} / \mathrm{L}$ $\mathrm{H}_{2} \mathrm{SO}_{4}$ ) becomes shifted negatively, its slope decreases and an obvious maximum appears (Figure 1). Under otherwise identical conditions the latter is more pronounced when faster dropping is applied.

In the mentioned solution, under the influence of $10 \mathrm{k} \Omega$ additional resistance, DPP gives a broad signal and a pronounced minimum. The broadening is obvious, irrespective of the applied timing parameters whereas the "deepest" extreme results from a "combination" of 


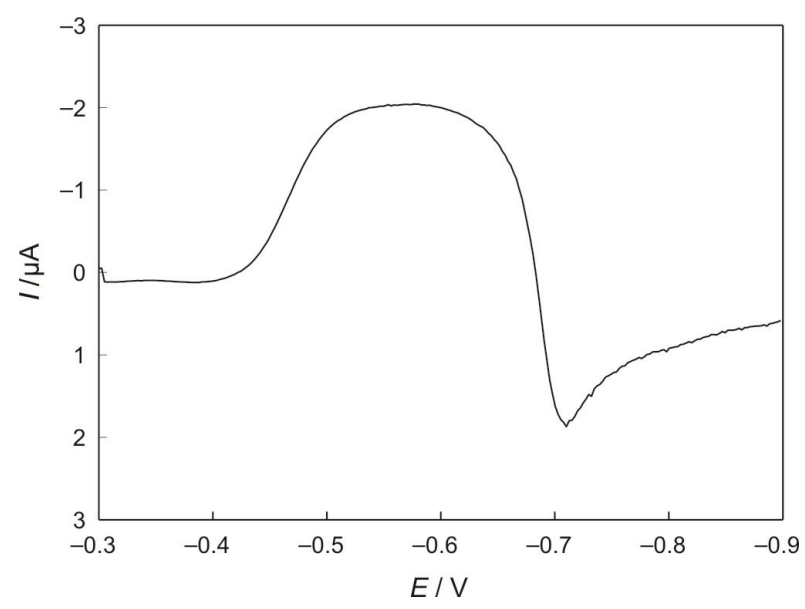

Figure 2. DPP response of the same solution as in Figure 1, obtained in the presence of inserted resistor $(10 \mathrm{k} \Omega)$. Drop time: $0.3 \mathrm{~s}$, pulse duration: $150 \mathrm{~ms}$, pulse height: $25 \mathrm{mV}$, scan increment: $2 \mathrm{mV}$, electrode: PAR 303, electrode surface: 1.68 $\mathrm{mm}^{2}$.

fast dropping and long pulses (Figure 2). Qualitatively, this result is in agreement with Figure 1, i.e. the fact that wave component of the net DPP response does not "see" $t_{\mathrm{d}}$ but the difference of $t_{\mathrm{d}}-t_{\mathrm{p}}$.

It is interesting that classical studies of polarographic maxima (obtained with simple DME), ${ }^{1}$ included measurements in the presence of added resistors. Under such conditions, so - called maxima of the first kind were reduced, whereas those of the second kind remained unchanged. In the present case, however, dc maximum and DPP minimum become more pronounced as the inner resistance increases. The effect does not depend on the polarographic analyzer and was confirmed by three different instruments.

DPP studies of $\mathrm{Cd}^{2+}$ reduction in nitrate, i.e. noncomplexing medium, influenced by added uncompensated resistance (up to $20 \mathrm{k} \Omega$ ) were described forty years ago. ${ }^{22}$ Combination of a long drop time $(2 \mathrm{~s})$, short pulses and relatively low metal concentrations (up to $0.02 \mathrm{mmol} / \mathrm{L}$ ) produced some effects that could be ascribed to an IR drop (i.e. to the difference between "official" and real potentials caused by the mentioned manipulation), but the current minimum did not appear, in accordance with our results.

Strictly speaking, measurements in the presence of added resistors are not fully equivalent to those performed at low electrolyte concentrations. ${ }^{23}$ Therefore,

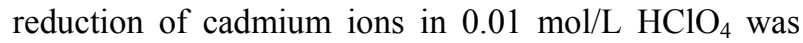
followed at different concentrations of dissolved metal. With usual potential and timing parameters $\left(t_{\mathrm{d}}=0.5 \mathrm{~s}\right.$, $t_{\mathrm{p}}=50 \mathrm{~ms}$, pulse height: $25 \mathrm{mV}$, step potential: $\left.2 \mathrm{mV}\right)$ it was not possible to obtain well defined DPP minimum or dc maximum (while using $663 \mathrm{VA}$ stand) even at a cadmium concentration of $1 \mathrm{mmol} / \mathrm{L}$, although some

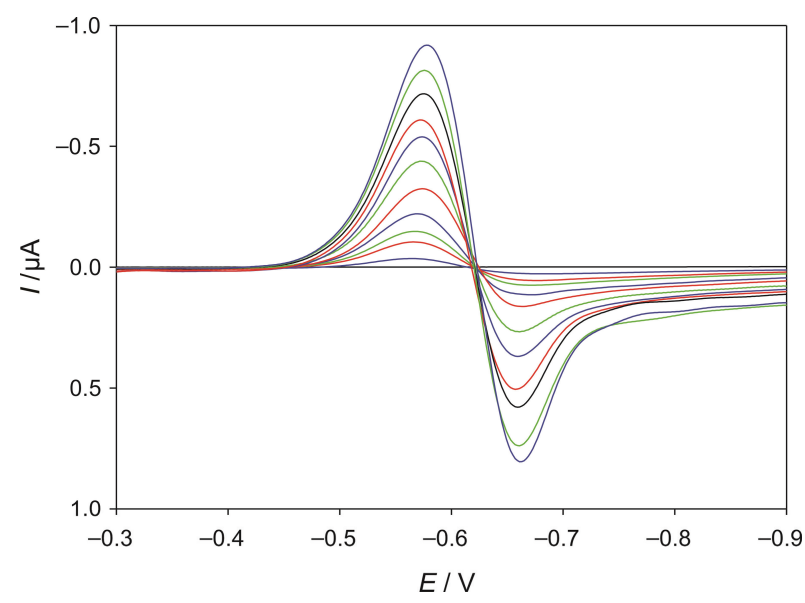

Figure 3. DPP response of $\mathrm{Eu}^{3+}$ in $0.01 \mathrm{~mol} / \mathrm{L} \mathrm{HClO}_{4}$. Measurements performed at the metal concentrations of (in the ascending order): $0,0.02,0.05,0.07,0.10,0.15,0.20,0.25$, $0.30,0.35,0.40$ and $0.45 \mathrm{mmol} / \mathrm{L}$ using large mercury drop $\left(0.53 \mathrm{~mm}^{2}\right)$ of $663 \mathrm{VA}$ stand. Other conditions include drop time of $0.5 \mathrm{~s}$, pulse duration of $50 \mathrm{~ms}$, pulse height of $50 \mathrm{mV}$ and step potential of $2 \mathrm{mV}$.

kind of poorly pronounced and "expanded" dc extreme appeared.

When $\mathrm{Eu}^{3+}$ reduction is followed instead, the DPP minimum appears even in relatively dilute solutions $\left(10^{-5} \mathrm{~mol} / \mathrm{L}\right)$. It increases with increasing metal concentration (Figure 3) but decreases and finally disappears after gradual addition of a concentrated sodium perchlorate solution. Such results are expected for an effect produced by the IR drop, although in the present case it stays questionable to what extent they are influenced by the fact that reduction rate depends on the electrolyte concentration. $^{24}$

It is known ${ }^{25}$ that errors produced by uncompensated ohmic resistance can be reduced in several different ways, one of which includes decreasing the size of the working electrode in order to minimize double layer capacity. From the practical point of view, it means that while working with the same electrode assembly and the same solution, results could be significantly changed by a simple increase/decrease of the mercury surface area (Figure 4).

Signals obtained with two different SMDE assemblies generally differ not only because of their different drop areas and cell geometries but also because of their characteristic drop formation times. ${ }^{26,27}$ For all those reasons we managed to obtain a well defined DPP minimum with $\mathrm{Eu}^{3+}$ in $0.01 \mathrm{~mol} / \mathrm{L} \mathrm{HClO}_{4}$ while using 663 VA stand (from Metrohm) but not under otherwise identical conditions, with the PAR 303 electrode, although in the latter case all three possible drop areas are bigger than one corresponding to the largest drop in the former. 


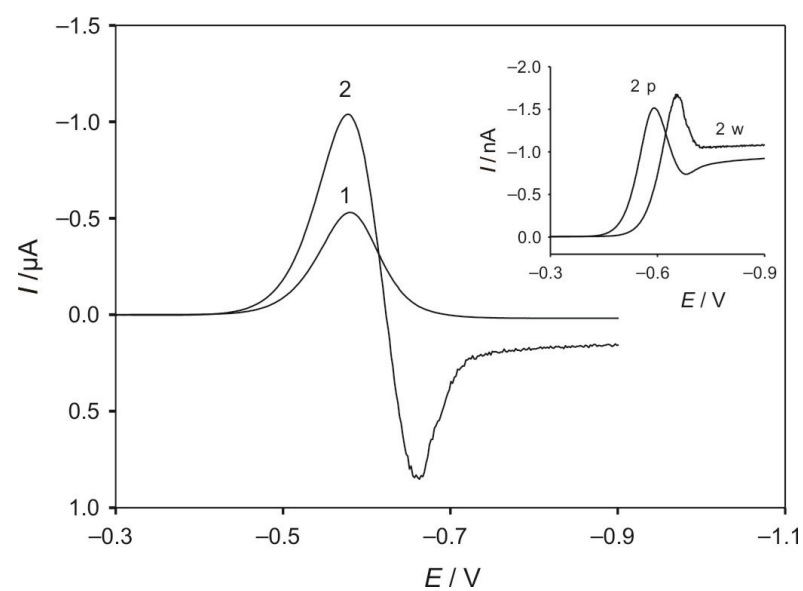

Figure 4. DPP signals of $0.5 \mathrm{mmol} / \mathrm{L} \mathrm{Eu}^{3+}$ in $0.01 \mathrm{~mol} / \mathrm{L}$ $\mathrm{HClO}_{4}$ recorded on (1) small $\left(0.26 \mathrm{~mm}^{2}\right)$ and (2) large $(0.53$ $\mathrm{mm}^{2}$ ) mercury drops of 663 VA stand using drop time of $0.5 \mathrm{~s}$, pulse duration of $50 \mathrm{~ms}$, pulse height of $50 \mathrm{mV}$ and step potential of $2 \mathrm{mV}$. Inset: wave $(2 \mathrm{w})$ and pulse (2p) components of signal 2.

From Figures 3 and 4, it follows that the absolute heights of the DPP minimum and reduction peak could be similar. Under such conditions, so-called wave and peak components of the net response are similar too. In other words, their names become inappropriate, although talking about forward and backward currents (as in square-wave voltammetry) does not seem much better.

\section{Reactant Adsorption}

Under the influence of the aforementioned results and those given in Ref. 17, investigation of cadmium(II) adsorption from iodide medium to the mercury electrode was performed using a carefully cleaned PAR 303 SMDE and concentrated electrolyte solutions in order to prevent or reduce possible influence of IR drop on experimental results. DPP reduction signals of the metal ion in acidified perchlorate medium were perfect irrespective of the applied timing parameters, but after addition of iodide ions, the well-known minimum appeared again. In this case it seemed to reflect adsorption of $\mathrm{CdI}_{2}$. Net DPP signals, obtained at different electrolyte, metal and ligand concentrations, were followed at varying timing parameters in attempt to find the origin of the current minimum and factors that make it more or less pronounced.

As in the situation with a significant IR drop, the minimum becomes more pronounced when the drop time decreases or pulse duration increases (Figure 5). In this case each minimum was treated as a kind of inverted peak measured from the baseline. Its area (instead of "deepness") presented as a function of the chosen timing parameter gives similar results as those presented in
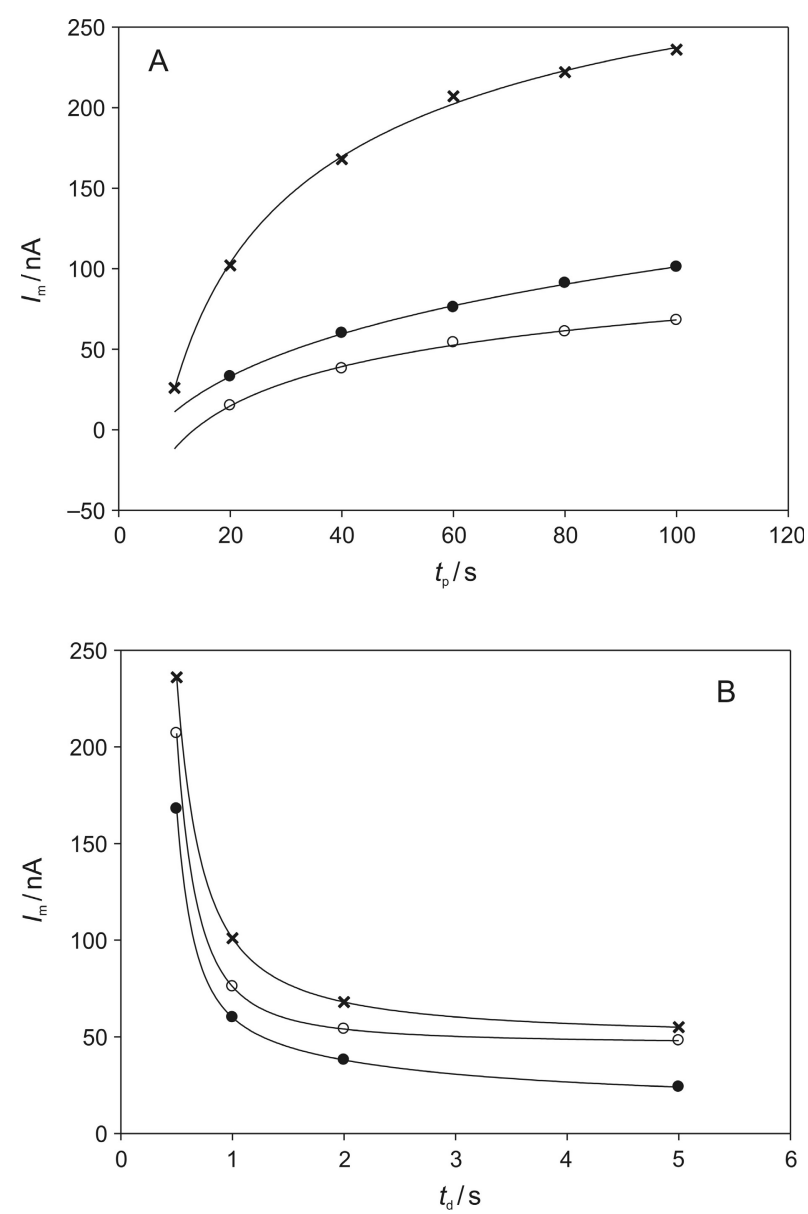

Figure 5. The influence of pulse duration (A) and drop time (B) on the "deepness" of DPP minimum. Solution composition: $0.2 \mathrm{mmol} / \mathrm{L} \mathrm{Cd}^{2+}, 15 \mathrm{mmol} / \mathrm{L} \mathrm{I}^{-}, 0.001 \mathrm{~mol} / \mathrm{L} \mathrm{HClO}_{4}$ at the ionic strength of $4 \mathrm{~mol} / \mathrm{L}$ achieved by $\mathrm{NaClO}_{4}$. Values given in (A) recorded at drop times of $0.5(\mathrm{x}), 1(\bullet)$ and $2 \mathrm{~s}(\mathrm{O})$. Values given in (B) recorded at pulse durations of $40(\bullet), 60(\circ)$ and $100 \mathrm{~ms}(\mathrm{x})$, pulse height of $25 \mathrm{mV}$ and scan increment of 2 $\mathrm{mV}$. Electrode: PAR 303, electrode surface area: $1.02 \mathrm{~mm}^{2}$.

Figure 5. Additionally, Figure 5B cannot be changed significantly if the difference $t_{\mathrm{d}}-t_{\mathrm{p}}$ is taken as an independent variable instead of $t_{\mathrm{d}}$.

The pronounced minimum appears in measurements on the large mercury drop, but becomes almost invisible when a small drop is applied instead under otherwise identical conditions. Taking into account the dependencies given in Figure 5, this result seems to reflect the importance of drop formation time which is significantly longer in the former than in the latter case. $^{26,27}$ The resulting apparent drop time by which the wave component is governed, decreases with increasing drop volume, producing so more pronounced current minimum.

For practical purposes it is important to find measuring conditions that prevent formation of any 


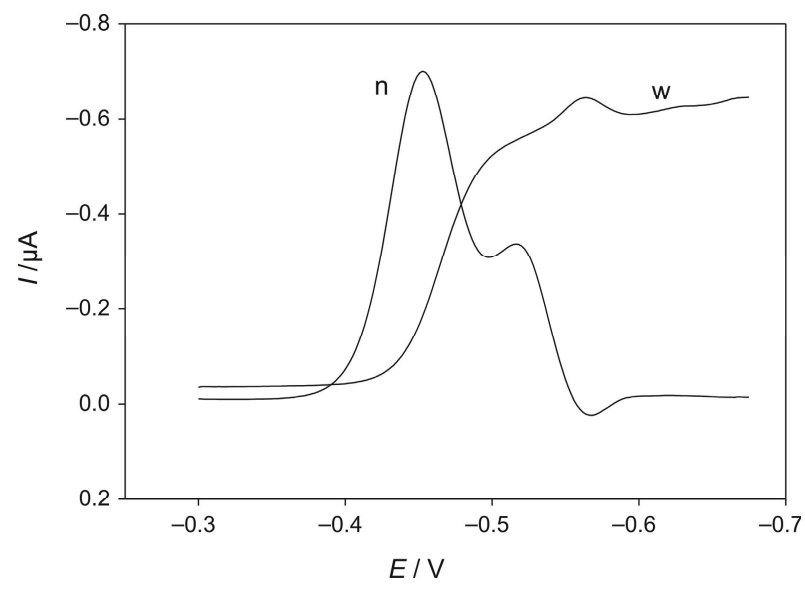

Figure 6. DPP minimum as a consequence of dc maximum at the ionic strength of $6 \mathrm{~mol} / \mathrm{L}$. Net response (n) and its wave component $(\mathrm{w})$, both recorded at drop time of $1 \mathrm{~s}$, pulse duration of $60 \mathrm{~ms}$, pulse height of $25 \mathrm{mV}$ and scan increment of 2 $\mathrm{mV}$ at the total dissolved cadmium and iodide concentrations of 0.22 and $4.8 \mathrm{mmol} / \mathrm{L}$, respectively. Electrode: PAR 303, drop surface: $1.02 \mathrm{~mm}^{2}$. (Pulse component omitted for clarity.)

extreme. Therefore, if not otherwise stated, all measurements in $\mathrm{Cd}^{2+}-\mathrm{I}^{-}$system were performed on a small mercury drop of a PAR 303 SMDE. Current minimum in DPP, however, appeared in many cases indicating the important role of other factors in its formation.

Going back to the origin of the DPP minimum, the previously mentioned de maximum can be confirmed as its "source" without difficulties. In Figure 6 the cadmium(II) reduction signal (at the ionic strength of $6 \mathrm{~mol} / \mathrm{L}$ and the highest possible relative concentration of $\mathrm{CdI}_{2}$ ) and its wave component are presented. It can be seen that both extremes (i.e. dc maximum and DPP minimum) appear in the same potential range and could be treated as peaks of similar dimensions, but different orientations. As a result of the way in which the final current $\left(I_{\text {net }}\right)$ is obtained (i.e. $\left.I_{\text {net }}=I_{\text {pulse }}-I_{\text {wave }}\right)$, the dc maximum becomes transformed to DPP minimum. The situation is not always so simple because pulse component, i.e. its shape and relative position with respect to the wave, sometimes also plays very important role. The most obvious "exceptions" of this type are examples with a deep DPP minimum and poorly pronounced $\mathrm{dc}$ maximum (Figure 7). In such a situation, minimum on the pulse component appears too, whereas the net minimum reflects the sum of both extremes, i.e. their absolute values. As far as we know, a minimum that appears on the pulse component has never been studied theoretically and/or experimentally. Therefore, conditions necessary for its formation remain unclear.

In addition to the timing parameters, composition of the electrolyte solution also plays an important role in formation of DPP adsorption minimum. When two different cadmium(II) concentrations are measured at an
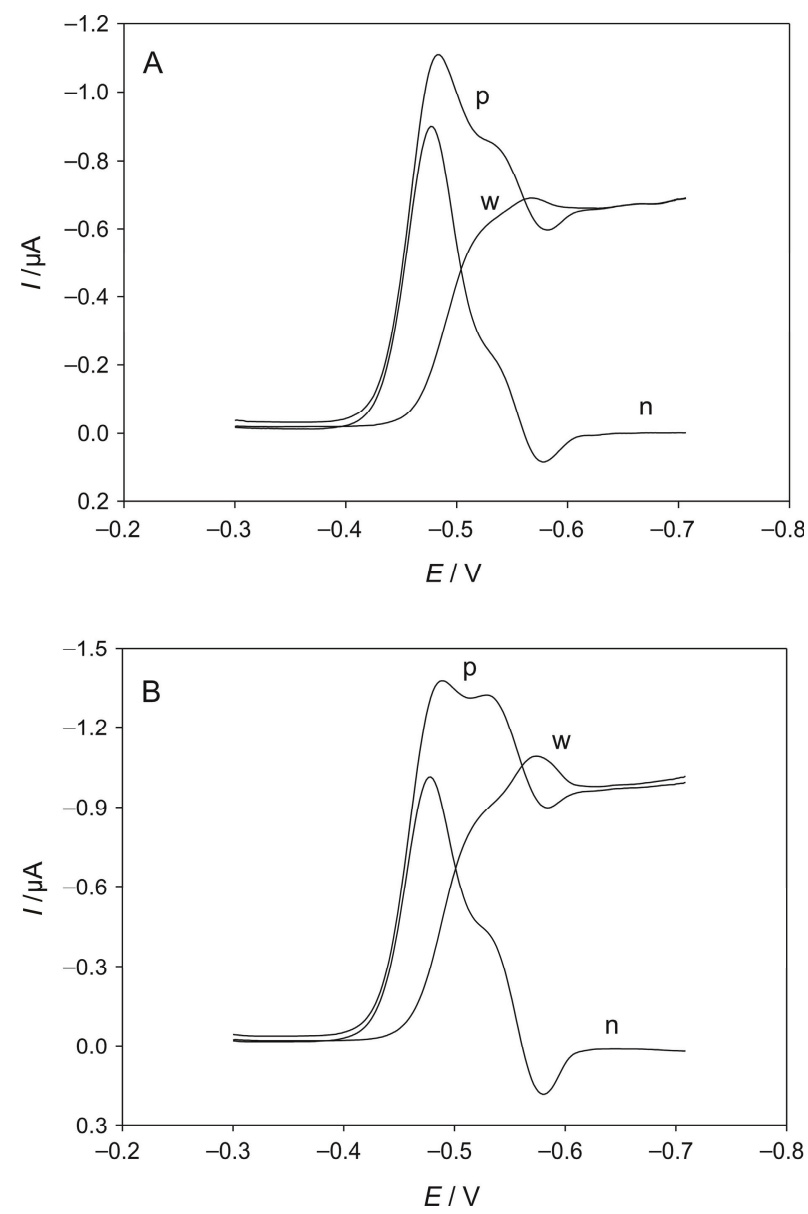

Figure 7. (A) DPP minimum that arises from the current minimum on the pulse component at the ionic strength of 4 $\mathrm{mol} / \mathrm{L}$. Net DPP signal and its components $(\mathrm{p}, \mathrm{w})$ recorded in solution containing $0.12 \mathrm{mmol} / \mathrm{L}$ cadmium(II) and $15 \mathrm{mmol} / \mathrm{L}$ iodide. Potential and timing parameters given in description of Figure 6. (B) The importance of both, maximum on the wave component (w) and minimum on the pulse component (p), in formation of minimum on the net DPP signal (n). Measurements performed with $0.22 \mathrm{mmol} / \mathrm{L}$ cadmium(II), $15 \mathrm{mmol} / \mathrm{L}$ iodide at the ionic strength of $4 \mathrm{~mol} / \mathrm{L}\left(t_{\mathrm{d}}=0.5 \mathrm{~s}, t_{\mathrm{p}}=40 \mathrm{~ms}\right.$, pulse height: $25 \mathrm{mV}$, step potential: $2 \mathrm{mV}$ ). Electrode: PAR 303, drop surface: $1.02 \mathrm{~mm}^{2}$.

ionic strength of $1 \mathrm{~mol} / \mathrm{L}$ and a constant iodide level $(0.05 \mathrm{~mol} / \mathrm{L})$ the extreme of interest stays unchanged although the net reduction signal reflects the increased reactant concentration. In more concentrated electrolyte solutions (ionic strength of 4 or $6 \mathrm{~mol} / \mathrm{L}$ ) however, the minimum is deeper at a higher metal concentrations, although the cadmium(II) level in all the cases was chosen so to produce complete coverage of the electrode surface by the surface active species $\mathrm{CdI}_{2}$.

Taking into account that three different ionic strengths (1, 4 and $6 \mathrm{~mol} / \mathrm{L})$ and three different iodide concentrations (50, 15 and $4.8 \mathrm{mmol} / \mathrm{L}$, respectively) were studied, it seems interesting to correlate DPP min- 


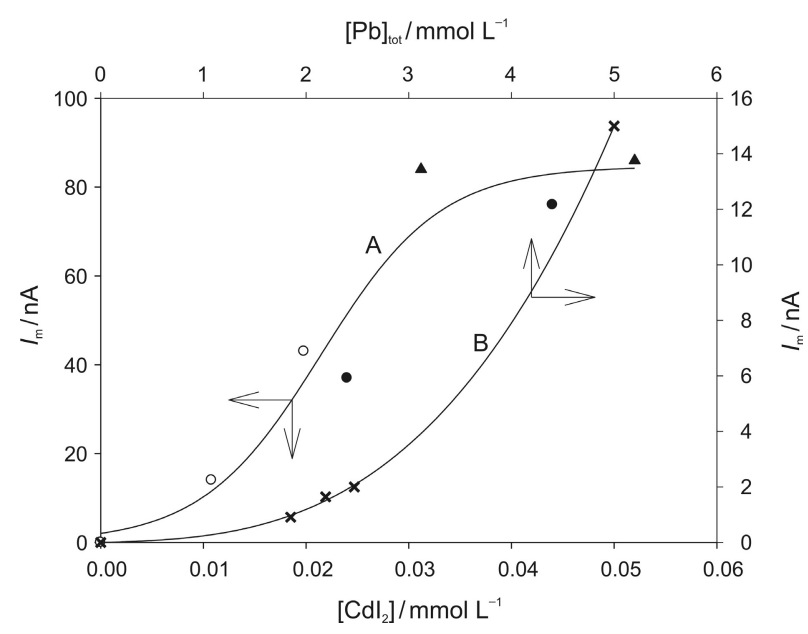

Figure 8. "Deepness" of the net DPP minimum in dependence on concentration of (A) dissolved $\mathrm{CdI}_{2}$ and (B) total dissolved lead(II). Measurements performed (A) at ionic strengths of 1 $(\Delta), 4(\bullet)$ and $6 \mathrm{~mol} / \mathrm{L}(\mathrm{O})$ and two different concentrations of total cadmium(II) applying potential and timing parameters given in description of Figure 6. Curve (B) shows experimental values obtained under the influence of about $3 \mathrm{k} \Omega$ inner resistance at ionic strength of $4 \mathrm{~mol} / \mathrm{L}$ (i.e. $2 \mathrm{~mol} / \mathrm{L}$ of $\mathrm{NaCl}+2 \mathrm{~mol} / \mathrm{L}$ of $\mathrm{NaClO}_{4}$ ) using drop time of $0.5 \mathrm{~s}$, pulse height of $24 \mathrm{mV}$ and step potential of $2 \mathrm{mV}$. Electrode: PAR 303, electrode surface: (A) 1.02 (B) $1.68 \mathrm{~mm}^{2}$.

imum with absolute concentration of the mentioned complex in the aqueous phase (calculated by means of the corresponding stability constants - taken from a data base $^{28}$ or evaluated from the existing values ${ }^{18}$ ). Although all the points, obtained at ionic strengths of 1 and $6 \mathrm{~mol} / \mathrm{L}$, seem to indicate nearly perfect sigmoidal dependence, the other two values (measured at the electrolyte concentration of $4 \mathrm{~mol} / \mathrm{L}$ ) are significantly lower (Figure 8, curve A). The effect cannot be explained in terms of data quality because standard error of repeated measurements is within $0.5 \%$. In addition to the species equilibria, the plot reflects some other changes produced by the increased electrolyte level such as decreased diffusion coefficients, increased surface concentration of free iodide ions, changed parameters of the adsorption isotherm etc. Therefore this type of presentation has perhaps more qualitative than quantitative importance.

Similar presentation of results obtained under the influence of significant resistance within the working electrode assembly (about $3 \mathrm{k} \Omega$ ) in $\mathrm{Pb}^{2+}-\mathrm{Cl}^{-}-\mathrm{ClO}_{4}{ }^{-}$ system, using electrolyte mixture of a constant composition ( $2 \mathrm{~mol} / \mathrm{L}$ of each anion) but gradually increasing the level of the total dissolved metal, gives the curve B in Figure 8, which does not reach the plateau. In other words, the pronounced minimum (irrespective of its origin) is formed at relatively high reactant concentrations, thus limiting it's possible application for practical purposes. The effect, which appears not only under the influence of potential drop, but also as a result of adsorption, is in agreement with the fact that increased (pseudo)capacity affects the final signal in the same way as decreased conductivity. ${ }^{22}$

\section{Literature Examples and Possible Applications}

Among older literature results on DPP minimum, the signal that reflects catalytic hydrogen evolution ${ }^{29}$ in Te(IV) solutions seems especially interesting. Currentconcentration linearity was observed, not only for the main reduction peak but also for the minimum. Moreover, possible measurement of the peak to peak current, i.e. use of the whole phenomenon for analytical purposes, was discussed.

Among newer examples we noticed an application of the effect in determination of polysulfides ${ }^{12}$ because of proportionality between analyte concentration and "deepness" of the DPP minimum. In that case however, the dc maximum could not be observed. On the contrary, some kind of minimum appeared on the limiting wave current, which couldn't be correlated with DPP minimum, located more positively, although the authors tried to do so. As follows from the discussion, given in previous paragraphs, the dc minimum is expected to appear as a (small) additional peak in the net DPP response. The DPP minimum could result from minimum on the plateau of the pulse component, especially when dc maximum is not pronounced. Therefore, the factors that cause formation of a minimum on the plateau of the pulse component and relationship between the latter and dc maximum should be identified. From such results, possible applications of DPP minimum in electro(analytical) chemistry would become clear. In this context, the conditions under which the minimum current is proportional to the concentration of an electroactive analyte should be identified too. As follows from the given examples, such systems are known. Our own results, however, point to a polynomial rather than linear dependence, i.e. to the existence of only limited concentration regions in which minimum current could be treated as proportional to the analyte concentration. At present, it seems that formation of the DPP minimum and/or dc maximum on polarograms obtained by the static mercury drop electrode could be used as an indication(s) of pronounced resistance within electrode system, poor conductivity of the measuring solution or adsorption of an electroactive reactant.

\section{CONCLUSION}

Analysis of the current minimum in differential pulse polarography (DPP) brought once again to view the abundance of information comprised in signals arising 
from complex excitation under potentiostatic conditions. A deeper analysis of the minimum in a net DPP signal, obtained under the influence of pronounced IR drop or reactant adsorption, revealed that the effect may originate from the maximum on a dc component, minimum on pulse component or both. The application of this information in characterization of electrode processes or recognition of solution composition is proposed, as well as further analyses of similar regularities in wave shapes that could reveal interesting information about the system under investigation. From the practical point of view, it is important to find if some other influences, in addition to reactant adsorption and IR drop, could produce a similar effect. New experiments with SMDE are needed as virtually all DPP current minima, described in the literature, were based on measurements with DME which seems to give somewhat different results. As far as we know, calculated signals of this type have never been obtained. For a full understanding of the whole phenomenon their "production" would be very useful.

Acknowledgements. Financial supports of the Ministry of Science, Education and Sports of the Republic of Croatia within the projects Electroanalytical Investigations of Microcrystals and Traces of Dissolved Compounds and Interactions of Trace Metal Species in an Aquatic Environment are gratefully acknowledged.

\section{REFERENCES}

1. J. Heyrovský and J. Kůta, Principles of Polarography, Publishing House of Czechoslovak Academy of Science, Prague 1966.

2. A. M. Bond, Modern Polarographic Methods in Analytical Chemistry, Dekker, New York 1980.

3. A. J. Bard and L. R. Faulkner, Electrochemical Methods, Second Edition, Wiley, New York, 2001.

4. J. J. Lingane and L. W. Niedrach, J. Am. Chem. Soc. 71 (1949)
196-204.

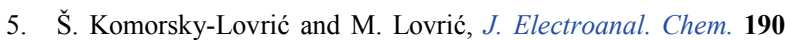
(1985) 1-20.

6. M. Lovrić and M. Zelić, J. Electroanal. Chem. 316 (1991) 315-328.

7. H. P. van Leeuwen, J. Buffle, and M. Lovrić, Pure Appl. Chem. 64 (1992) 1015-1028.

8. H. H. Bauer, Electrochim. Acta 18 (1973) 355-358.

9. A. N. Frumkin, N. V. Fedorovich, B. B. Damaskin, E. V. Stenina, and V. S. Krylov, J. Electroanal. Chem. 50 (1974) 103-111.

10. M. Noufi, C. Yarnitzky, and M. Ariel, Electroanalysis 8 (1996) 836-841.

11. M. Lovrić, J. Electroanal. Chem. 465 (1999) 30-36.

12. S. Kariuki, M. J. Morra, K. J. Umiker, and I. F. Cheng, Anal. Chim. Acta 442 (2001) 277-285.

13. R. de Levie, J. Electroanal. Chem. 552 (2003) 223-229.

14. M. Kopanica and V. Stará, J. Electroanal. Chem. 98 (1979) 213-221.

15. J. E. Anderson and A. M. Bond, Anal. Chem. 52 (1980) 1439-1445.

16. M. Zelić, I. Pižeta, and M. Branica, Anal. Chim. Acta 281 (1993) 63-70.

17. I. Pižeta and M. Zelić, Anal. Chim. Acta 319 (1996) 31-37.

18. M. Zelić and M. Lovrić, J. Electroanal. Chem. 541 (2003) 67-76.

19. I. Pižeta, M. Lovrić, M. Zelić, and M. Branica, J. Electroanal. Chem. 318 (1991) 25-38.

20. I. Ružić, J. Electroanal. Chem. 75 (1977) 25-44.

21. M. Zelić and D. Jadreško, Electroanalysis 20 (2008) 1897-1902.

22. J. B. Flanagan, K. Takashi, and F. C. Anson, J. Electroanal. Chem. 81 (1997) 261-273.

23. W. B. Schaap and P. S. McKinney, Anal. Chem. 36 (1964) 29-35.

24. M. Zelić, Croat. Chem. Acta 76 (2003) 241-248 and references therein.

25. www. ecochemie.nl/Applications/

26. W. M. Peterson, Am. Lab. 11 (1979) 69-78.

27. D. Omanović and M. Branica, J. Electroanal. Chem. 543 (2003) 83-92.

28. NIST Critically Selected Stability Constants of Metal Complexes, Version 5.0, NIST Standard Reference Data, Gaithersburg 1998.

29. K. Hasebe, Bul. Chem. Soc. Japan 52 (1979) 1056-1059. 